

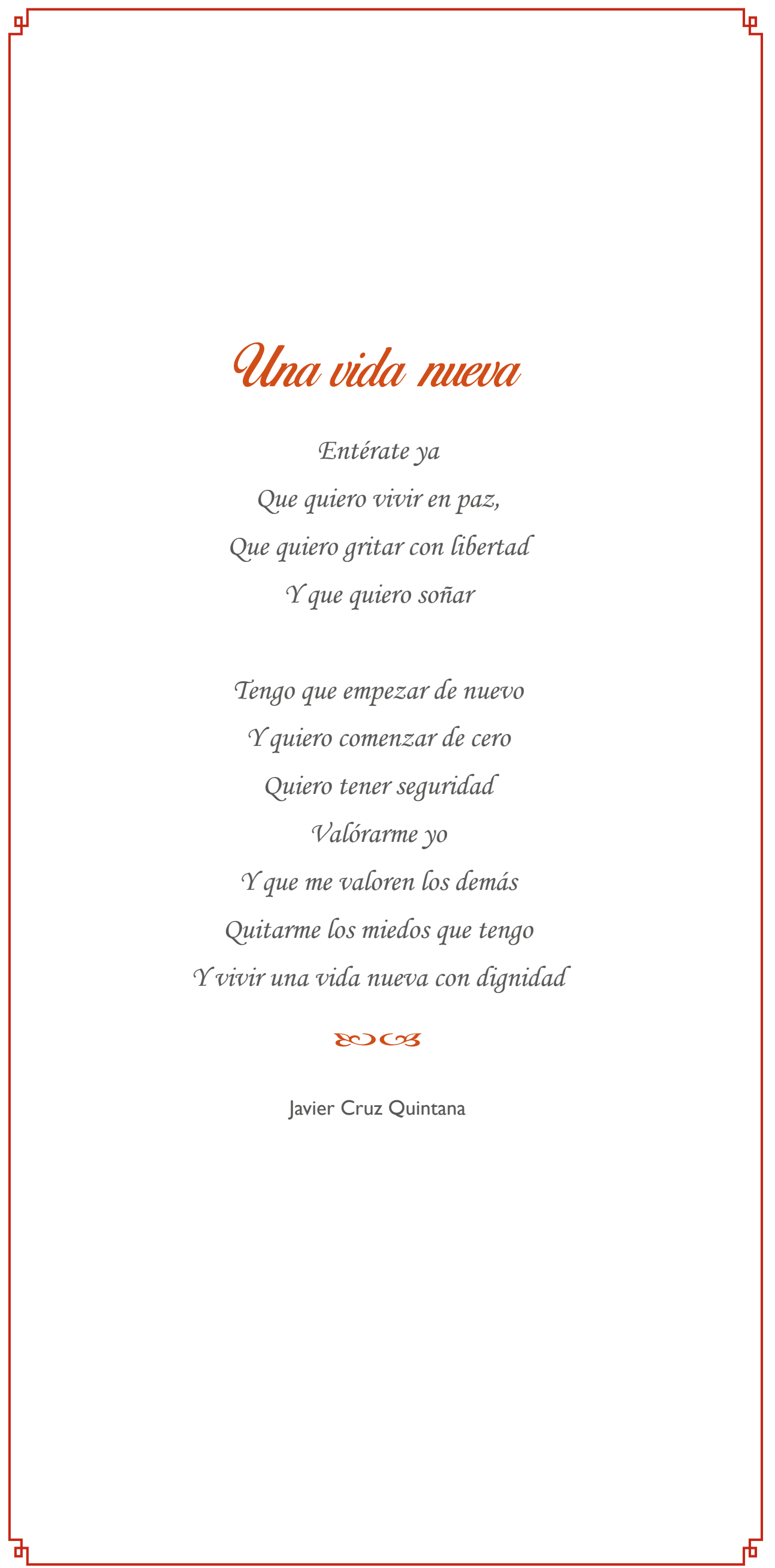




\title{
6. La violencia en países del Triángulo Norte de Centroamérica (TNCA). Análisis retrospectivo, 1999-2015
}

\author{
Marco Vinicio Benavides Osorto ${ }^{1}$ \\ Recibido 23-03-20I 7 / Aceptado 08-05-2017
}

\begin{abstract}
RESUMEN: En este artículo trata de la violencia del TNCA (Triángulo Norte de Centroamérica), con las tasas de homicidios intencionales más altas del planeta, siendo en el 2015, El Salvador (83,5), Honduras (57), Guatemala (34.9), afectados en su orden por altos índices de violencia. En los tres casos, las cifras sobrepasan el promedio mundial de 6.2 por cada 100 mil habitantes estipulado por la Organización Mundial de la Salud. Por su parte la ONU define la región como la más mortífera del mundo, donde uno de cada 50 hombres morirá asesinado antes de los $3 I$ años. El Triángulo Norte de Centroamérica (TNCA) -Guatemala, Honduras y El Salvador- cuenta con una población aproximada de 31 millones de habitantes y 242.4 mil kilómetros cuadrados, lidera los índices de migración y criminalidad en la región, las altas tasas de homicidios, solo se comparan con zonas en guerra como Siria y Afganistán, países que están por encima del umbral de los 10 homicidios por cada 100,000 habitantes, situación que la Organización Mundial de la Salud (OMS) considera como un problema epidémico. La metodología empleada es un análisis de tipo descriptivo, transversal, comparativo durante los años 1999-2015. En el periodo 1999- 2015 en los países delTNCA fallecieron por homicidio violento un total de 21 7,333 personas. Se compararon datos entre países de Triángulo Norte, durante los últimos 17 años, se tomó en cuenta los homicidios intencionales reportados por las diversas agencias gubernamentales de cada país. La fuente de datos consultados fue: INACIF (Guatemala), COEPOL (Honduras), IML (El Salvador). La situación de violencia en la región ha sido catalogada como un fenómeno multicausal, atribuyen la creciente violencia a la influencia del narcotráfico, altos niveles de corrupción, violencia intrafamiliar, organización eficiente de grupos delictivos, factores que contribuyen al aumento y profundización de la delincuencia y el crimen organizado, que se reflejan en estructuras militares, estructuras políticas y estructuras sociales. Las maras y pandillas constituyen un factor que contribuye al incremento de la violencia al servicio al tráfico de drogas y de personas que contratan sus servicios como sicarios, distribución y venta de droga, lo que ha provocado el aumento del consumo local y de la conflictividad social en los tres países.
\end{abstract}

Palabras claves: TNCA (Triángulo Norte de Centroamérica), tasas de homicidios, metodología, situación de violencia, maras y pandillas.

ABSTRACT: This article tries to the violence of the TNCA (Northern Triangle of Central America), with the highest rates of intentional homicides on the planet, being in 2015, El Salvador (83.5), Honduras (57), Guatemala (34.9), in its order affected by high rates of violence. In all three cases, the figures exceed the world average of 6.2 per 100 thousand inhabitants, as stipulated by the World Health Organization. Meanwhile the UN defines the region as the most deadly in the world, where one of every 50 men die murdered before the age of 3 I.Triangle North of Central America (TNCA) - Guatemala, Honduras and El Salvador - has a population of about 31 million inhabitants and 242.4 thousand square kilometers, leads the levels of migration and crime in the region, the high homicide rates, only compared with areas in war as Syria and Afghanistan, countries that are above the threshold of 10 homicides per 100,000 inhabitants situation that the World Health Organization (who) considers as an epidemic problem. The methodology used is a descriptive, transversal, comparative analysis during the years 1999-2015. In the period 1999-20I5 at the TNCA countries died of violent homicide a total of 21 7,333 people. Comparing data between northern triangle countries, during the past 17 years, took into account the intentional homicides reported by various government agencies in each country. The source of data consulted was: was (Guatemala), COEPOL (Honduras), IML (El Salvador). The situation of violence in the region has been classified as a multicausal phenomenon, attributed the increasing violence to the influence of drug trafficking, high levels of corruption, violence, efficient organization of criminal groups, factors contributing to the increase and deepening of crime and organized crime, which are reflected in military structures, political structures and social structures. The maras and gangs are a contributing factor to the increase in violence to the service to the trafficking of drugs and people who hire their services as hired killers, distribution and sale of drugs, which has led to the increase of local consumption and the social conflicts in the three countries.

Keywords: TNCA (triangle North of Central America), rates of homicide, methodology, situation of violence, maras and gangs.

\section{Introducción}

El Triángulo Norte de Centroamérica (TNCA) -Guatemala, Honduras y El Salvador- cuenta con una población aproximada de 31 millones de habitantes y 242.4 mil kilómetros cuadrados, lidera los índices de migración y criminalidad en la región, las altas tasas de homicidios, solo se comparan con zonas en guerra como Siria y Afganistán, países que están por encima del umbral de los 10 ho- micidios por cada 100,000 habitantes, situación que la Organización Mundial de la Salud (OMS) ha considerado como un problema epidémico.

De acuerdo a la Oficina de Naciones Unidas contra la Droga y el Delito (UNODC), el Instituto Transnacional (TNI) y la fundación Friedrich-Ebert-Stiftung en Colombia (FESCOL), la situación de violencia en la región ha sido catalogada como un fenómeno multicausal, quienes atribuyen la creciente violencia a la influencia del narcotráfico, altos

\footnotetext{
${ }^{1}$ Máster en Demografía y Desarrollo, UNAH. Dr. En Medicina y Cirugía, UNAH, correo electrónico:mvbo1961@yahoo.es.
} 
niveles de corrupción, violencia intrafamiliar, organización eficiente de grupos delictivos, factores que contribuyen al aumento y profundización de la delincuencia y el crimen organizado reflejándose en las estructuras militares, estructuras políticas y estructuras sociales. Por su parte la ONU define la región como la más mortífera del mundo, donde uno de cada 50 personas morirá asesinado antes de los 31 años.

Estos niveles de crimen y violencia a través del Triángulo Norte no se produjeron en el vacío: son alimentados por una profunda inseguridad económica, pues en estos países la pobreza a un alto porcentaje de su población, además sus oportunidades educativas son limitadas lo que incide negativamente en su inserción laboral que en gran parte de las ocasiones se realiza en condiciones de informalidad y precariedad laboral.

Por otra parte es evidente que la judicatura y el poder judicial es disfuncional en los países del Triángulo Norte esto ha contribuido a incrementar los niveles del crimen y violencia, de la misma manera incide en el desarrollo económico sostenible y la competitividad, situación que afecta a la población que día a día mediante trabajo y esfuerzo busca sobrevivir y en el mejor de los casos salir de la pobreza y el subdesarrollo.

\section{Metodología}

En el desarrollo de este artículo se realizó un análisis de tipo descriptivo, transversal y comparativo; se consideró como unidad de análisis la población violentada por homicidio intencional. Se calcularon las tasas estandarizadas de mortalidad por homicidios tanto a nivel nacional como a nivel de países del Triángulo Norte.

\begin{tabular}{|c|}
\hline Tasa de Homicidios intencionales: \\
$\begin{array}{c}\text { Defunciones registradas por homicidios intencionales, } \\
\text { año } X(\text { por 100,000h) } \\
\text { Población nacional a mitad del año }\end{array}$
\end{tabular}

Se utilizó como referente la población nacional total por año 1999-2015, hasta octubre 2016.

Se compararon datos entre países de Triángulo Norte, durante 17 años, se tomó en cuenta los homicidios intencionales reportados por las diversas agencias gubernamentales de cada país. La fuente de datos consultados fue: INACIF (Guatemala), COEPOL (Honduras), IML (EI Salvador).

En cuanto a homicidios intencionales, se recalca que los datos difieren por uno o dos puntos, esto porque al consultar con diversos medios estadísticos, hay datos numéricos levemente alterados, al igual que los datos de población para un año especifico pueden variar, pese a ello el resultado estadístico demográfico permite apreciar el problema.

\section{Discusión de resultados}

\subsection{Victimas con necesidad de protección}

En mayor medida los grupos más vulnerables a los efectos de la violencia son los habitantes de los barrios que se caracterizan por el limitado acceso a servicios e infraestructura básica, lo que refleja desigualdad, inequidad y exclusión social, unido a la inseguridad ciudadana que se percibe en todo el país.

Las principales víctimas de la muerte son hombres jóvenes, con frecuencia víctimas y perpetradores, que mueren abatidos por armas letales antes de que logren llegar los servicios de emergencia al lugar del hecho, según la ACAPS (2014) a la par de los homicidios se desarrollan otras situaciones de violencia (OSV), entre las que destacan:

Los niños y niñas que nacen y crecen en este entorno opresivo de muerte y agresión, con limitadas oportunidades de acceso al estudio, salud y desarrollo.

W Las mujeres, victimas invisibles de violencia dentro y fuera de sus hogares, las cuales son expuestas a altos niveles de violencia sexual y abusos físicos y psicológicos.

Las personas que se ven forzadas a desplazarse dentro del país o de forma transfronteriza, abandonando sus raíces, familias en busca de un nuevo destino; grupo que cada vez incluye más menores no acompañados y están en gran riesgo y necesidad de protección.

Las familias que pierden sus medios de vida o a la principal persona generadora de ingresos. También aqueIlas familias cuyos miembros productivos son heridos de gravedad o discapacitados como consecuencia de OSV y tienen que enfrentarse a gastos médicos.

W Las personas mayores, discapacitadas, población LGBTI y enfermos crónicos que viven en zonas con altos niveles de violencia y control territorial también son vulnerables.

Todas estas víctimas parecen invisibles para los órganos del Estado, y como tal no han sido objeto de justicia, ni 
han sido priorizados por la institucionalidad vigente. En este contexto se hace necesario elaborar los perfiles de las personas con mayor vulnerabilidad frente a la criminalidad y la violencia para entender mejor sus necesidades e implementar programas de desarrollo que generen estructuras de cambio en la vida de esta población.

\subsection{Poblaciones, países con homicidios cuantificados en el Triángulo Norte}

\section{El Salvador}

En el 2015, El Salvador clasificó como el país más peligroso del Hemisferio Occidental, con una tasa de homicidio de 108,5 homicidios por cada 100.00 personas. Una comparación entre El Salvador y USA, permite comparar que El Salvador, es 24 veces más peligroso que los Estados Unidos, pues este último, tuvo una tasa de homicidio de 4,5 por cada 100.000 personas en el 2014 (Gráfico $\left.\mathrm{N}^{\circ} 1.\right)$

La violencia es todavía peor en ciertos departamentos de dicho país, por ejemplo, San Salvador, la capital de El Salvador, tenía un promedio de un asesinato cada hora durante agosto del 2015 - el número más alto de asesinatos desde el fin de la horrífica guerra civil en el 1992.

Gráfico № 1: Homicidios por cada $100,000 /$ hab, en países del Triángulo Norte de C.A y USA, durante el año 2015

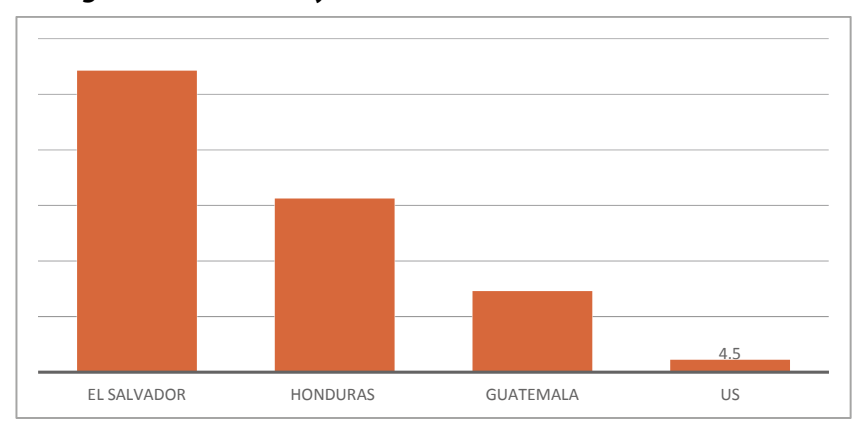

Fuente. Elaboración propia en base a datos (20/5)

INACIF (Guatemala), COEPOL (Honduras), IML (El Salvador).

En 2015 en El Salvador las rivalidades entre las dos principales pandillas del país, la MS13 y Barrio 18, además de las tácticas de gobierno como mano dura policial, contribuyeron a esta explosión de violencia. Sin embargo, en realidad los niveles de homicidios comenzaron a dispararse durante la segunda mitad de 2014, luego el fracaso de la tregua entre pandillas que inició en 2012, entre estas dos pandillas (Cuadro $\mathrm{N}^{\circ} 1$ ).

Tan solo en un año del 2014-2015, los homicidios aumentaron en 2,719 personas asesinadas (69\%), que es la diferencia del año 2014 (3,942), con el año 2015 $(6,661)$, todo esto se debe a la ruptura del pacto de no agresión suscrito por el gobierno del expresidente Fúnez con las maras, de alguna manera el aumento de los homicidios se debe a la no renovación de dicho pacto por el actual presidente Sánchez Cerén, de no dar cabida a la impunidad.

Cuadro $N^{\circ}$ 1. Homicidios en El Salvador, 1999-2015

\begin{tabular}{|c|c|c|}
\hline Año & El Salvador & Tasa de homicidio \\
\hline 1999 & 2,270 & 36.9 \\
\hline 2000 & 2,341 & 37.3 \\
\hline 2001 & 2,349 & 39.7 \\
\hline 2002 & 2,163 & 36.3 \\
\hline 2003 & 2,195 & 36.7 \\
\hline 2004 & 2,932 & 48.7 \\
\hline 2005 & 3,812 & 55.4 \\
\hline 2006 & 3,928 & 64.7 \\
\hline 2007 & 3,497 & 58.1 \\
\hline 2008 & 3,179 & 52.0 \\
\hline 2009 & 4,382 & 71.3 \\
\hline 2010 & 4,004 & 53.8 \\
\hline 2011 & 4,371 & 70.5 \\
\hline 2012 & 2,594 & 41.7 \\
\hline 2013 & 2,490 & 39.9 \\
\hline 2014 & 3,942 & 62.8 \\
\hline 2015 & 6,661 & 83.5 \\
\hline
\end{tabular}

Fuente. Elaboración propia en base a datos de IML, El Salvador

Se recalca que los muertos durante estos 17 años (1999-2015), ascienden a 57,110 personas abatidas por violencia, es decir homicidios intencionales (Grafico $\mathrm{N}^{\circ} 2$, Cuadro $\mathrm{N}^{\circ} 1$ ). Estadísticamente se espera que los homicidios por violencia irán en aumento, especialmente sino se desarrollan políticas judiciales, legislativas, económicas, educativas, de inclusión, combate frontal al crimen organizado y las pandillas, así como oportunidades para que las personas tengan el mínimum vital, que les permita vivir dignamente.

En El Salvador, el gobierno ha declarado repetidamente que el aumento de la violencia de las maras constituye la mayor amenaza a la que el país se ha enfrentado en decenios, y ha creado nuevos marcos de orden jurídico, militar y de aplicación de la ley para hacerle frente.

La pobreza, la falta de empleo y la separación de familias no son un fenómeno nuevo en El Salvador. Lo que sí es 
nuevo es el aumento de la tasa de homicidio. El fuerte aumento de la migración y las solicitudes de asilo está directamente relacionado con este fenómeno.

El gobierno salvadoreño se ha negado a establecer esa conexión, y en su lugar se ha enzarzado en una serie de ambigüedades respecto a las amenazas a la seguridad a las que, según afirma, enfrenta el país

\section{Gráfico №2. Homicidios en El Salvador 1998-2015}

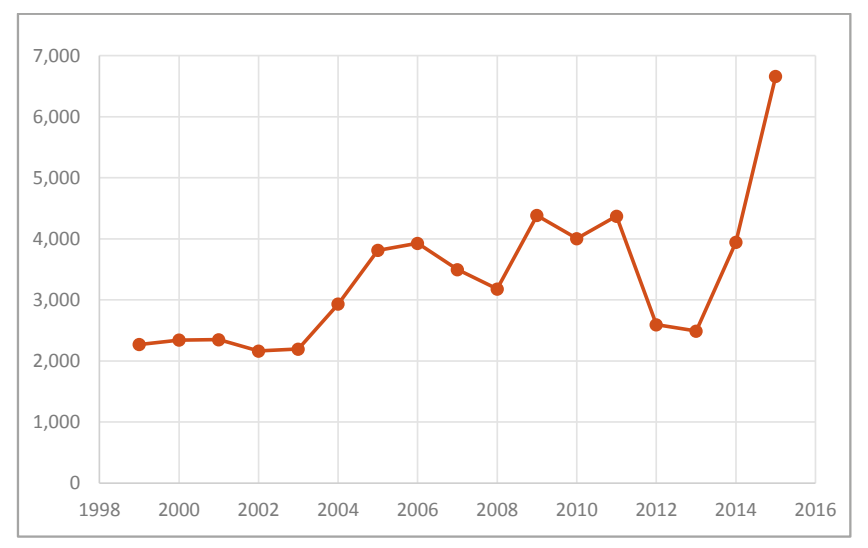

Fuente. Elaboración propia en base a datos IML (El Salvador)

\section{Honduras}

Las cifras de la policía muestran que los homicidios han disminuido en Honduras, país que antes era considerado la capital mundial del crimen. La tasa de homicidio en el 2015 en Honduras alcanzó a 57.0 homicidios por cada 100.00 personas, reflejando una disminución respecto al periodo 2009- 2014 (Gráfico No 3, Cuadro $\mathrm{N}^{\circ} 2$ ).

Aunque se ha cuestionado la validez de las estadísticas de homicidios divulgadas por el gobierno de Honduras, la organización civil Alianza por la Paz y la Justicia (APJ) acepta los datos de homicidio otorgados por el gobierno para el año 2015.

En particular, los casos de homicidios múltiples, que están estrechamente asociados con la violencia relacionada con el crimen organizado, aumentaron en 2015, a pesar de que la tasa de asesinatos disminuye.

El repunte máximo de homicidios se da en el año 2012, con leve disminución en los años posteriores. La sumatoria de muertes desde 1999 a octubre 2016, totalizan 78,182 persona asesinadas, que divididas entre 18 años promedian 4,343 persona asesinadas intencionalmente cada año, lo que equivaldría a 12 personas/día muertas de forma violenta cada día, lo que equivale a una muerte cada 2 horas, en el periodo de estudio.
En Honduras, el presidente Juan Orlando Hernández ha declarado que se encuentra trabajando por hacer del país un lugar más seguro. Sin embargo, la retórica de todas las secretarias de gobierno está clara: ahora que las tasas de homicidio han bajado, la violencia ya no es la razón principal para que la gente abandone el país. Sin embargo, la tasa de homicidio es un indicador incompleto en contextos en los que la violencia puede expresarse de muchas formas diferentes. Unido a ello la percepción de seguridad entre la población no ha aumentado lejos de ello, hay un temor generalizado a ser víctima de la violencia.

Cuadro № 2. Homicidios en Honduras, 1999-2015

\begin{tabular}{|c|c|c|}
\hline Año & Honduras & Tasa de homicidio \\
\hline 1999 & 2,563 & .6 \\
\hline 2000 & 3,176 & 49.0 \\
\hline 2001 & 3,488 & 53.4 \\
\hline 2002 & 3,629 & 54.2 \\
\hline 2003 & 2,224 & 32.4 \\
\hline 2004 & 2,155 & 30.7 \\
\hline 2005 & 2,417 & 32.9 \\
\hline 2006 & 3,018 & 41.0 \\
\hline 2007 & 3,574 & 47.4 \\
\hline 2008 & 4,473 & 58.0 \\
\hline 2009 & 5,280 & 67.0 \\
\hline 2010 & 6,236 & 76.0 \\
\hline 2011 & 7,104 & 86.5 \\
\hline 2012 & 7,172 & 85.5 \\
\hline 2013 & 6,427 & 75.1 \\
\hline 2014 & 5,802 & 66.5 \\
\hline 2015 & 5,148 & 57.0 \\
\hline
\end{tabular}

Fuente. Elaboración propia en base a datos COEPOL -Honduras

Gráfico № ${ }^{0}$. Homicidios en Honduras, 1999-2015

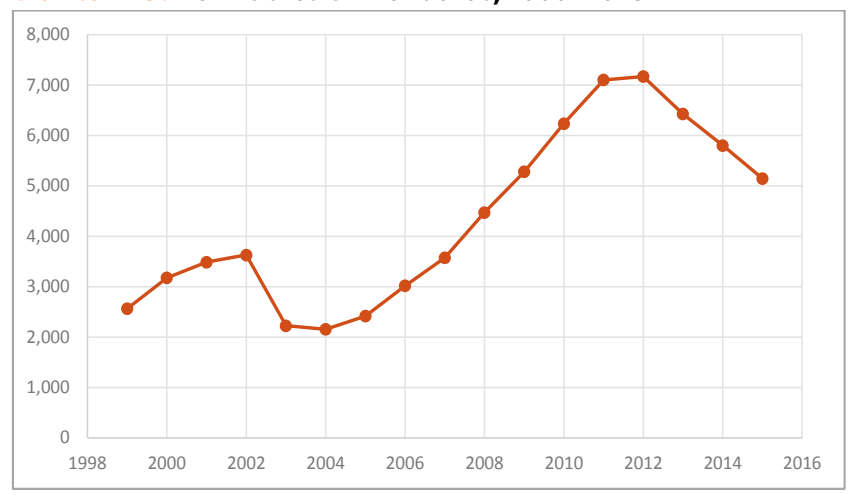

Fuente. Elaboración propia en base a datos de COEPOL Honduras 
En estos entornos, la incapacidad de hacer frente a una extorsión o la amenaza de perder la vida de un hijo o hija o familiar cercano en manos de la delincuencia son razones igual de importantes para huir del país. Aun cuando el desplazamiento interno predomina, puede ser sólo un precursor de una migración forzada, ya que la reubicación nacional no es siempre una alternativa viable en países que son geográficamente pequeños y están invadidos por las bandas organizadas.

Los cinco departamentos de Honduras que tuvieron las tasas de homicidio más altas en 2015 fueron: Cortés, Atlántida, Yoro, Francisco Morazán y Colón. Cuatros de estos departamentos se encuentran entre los que reciben mayor número de personas deportadas, lo que sugiere cierto traslape entre quienes huyen del país y quienes viven en zonas muy violentas.

\section{Muatemala}

En 2015, en Guatemala se registró 4.778 homicidios, lo que da como resultado una tasa de homicidios de 29,5 por cada 100,000 habitantes (Cuadro № 3, Gráfico № 4). Esto representa una leve disminución con respecto a los 4.998 homicidios registrados en 2014, y una disminución significativa con respecto al número de asesinatos en 2009, cuando el país registró una tasa de homicidios de 46,5 por cada 100.000 habitantes, el descenso de la tasa de homicidios refleja en alguna medida la disminución de la violencia por homicidio en este país.

El repunte máximo de violencia homicida intencional se da en el año 2009, con 6,498 asesinatos, 2008 con 6,292 , y otro repunte en el 2012 con 6,025 asesinados, el 2013 con 6,702 , un aumento de 47 fallecidos, respecto al año anterior, los años 2014 y 2015, muestran un leve descenso de homicidio.

En 18 años, se han contabilizado desde 1999-octubre, la suma de 90,914 fallecidas, que promedian 5,051 personas que han sido asesinadas cada año, diríamos 421 personas cada mes, un promedio de 18 personas por día.

\section{Homicidios totales en TNCA. 1999-2015}

En el periodo 1999- 2015 en los países del TNCA fallecieron por homicidio violento un total de 217,333 personas, el mayor aporte lo tuvo Guatemala con $40 \%$ de los casos, un segundo lugar lo ocupó Honduras con 34\% y el tercer lugar fue para El Salvador con $26 \%$. En otras palabras, en promedio en un periodo de 17 años la cantidad de personas muertas por homicidio aumento 2.3 veces, es decir una tasa de crecimiento de 1.4 (Gráfico $N^{\circ}$ 5).
Cuadro № 3. Homicidios en Guatemala. 1991-2015

\begin{tabular}{|c|c|c|}
\hline Año & Guatemala & Tasa de homicidio \\
\hline 1999 & 2,655 & 24.0 \\
\hline 2000 & 2,904 & 25.5 \\
\hline 2001 & 3,230 & 28.0 \\
\hline 2002 & 3,630 & 30.6 \\
\hline 2003 & 4,237 & 34.8 \\
\hline 2004 & 4,507 & 36.2 \\
\hline 2005 & 5,338 & 41.2 \\
\hline 2006 & 5,885 & 45.0 \\
\hline 2007 & 5,781 & 43.2 \\
\hline 2008 & 6,292 & 46.0 \\
\hline 2009 & 6,498 & 46.4 \\
\hline 2010 & 5,960 & 40.7 \\
\hline 2011 & 5,681 & 38.8 \\
\hline 2012 & 6,025 & 40.2 \\
\hline 2013 & 6,072 & 39.7 \\
\hline 2014 & 5,924 & 38.0 \\
\hline 2015 & 5,718 & 34.9 \\
\hline
\end{tabular}

Fuente. Elaboración propia en base a datos INACIF (Guatemala)

Gráfico № 4. Homicidios en Guatemala. 1991-2015

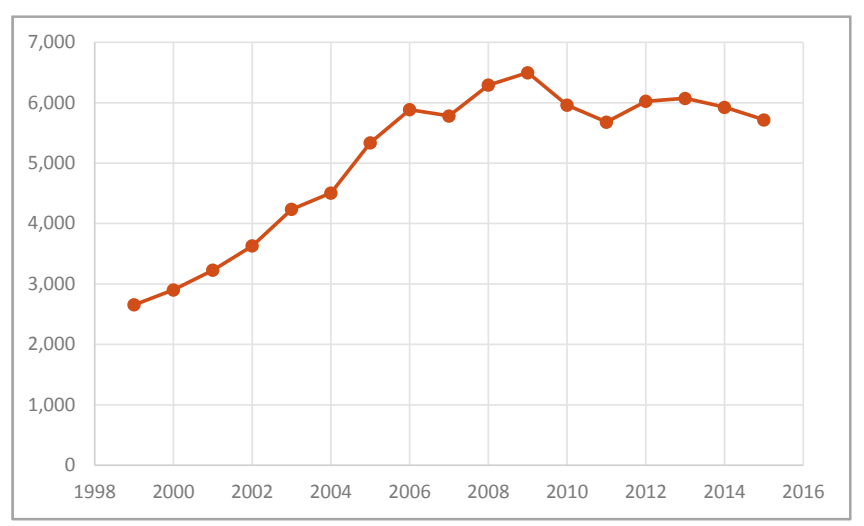

Fuente. Elaboración propia en base a datos INACIF-Guatemala.

La mayoría de esos homicidios se comenten con armas de fuego y gran parte de los mismos están relacionados con maras y pandillas y actividades relacionadas con el narcotráfico.

Las diferencias de seguridad pueden ayudar a explicar por qué las de los países del Triángulo Norte están huyendo no solamente hacía los Estados Unidos, también a los países vecinos e incluso países de Europa.

Entre 2008 y 2014, la ONU documentó un aumento en aplicaciones de asilo salvadoreñas, hondureñas, y guatemaltecas en México, Panamá, Nicaragua, Costa Rica, y Belice. 
Desde el comienzo del año 2014, más de 120.000 menores sin acompañantes y otras 120.000 más seres humanos, en grupos de familias, usualmente madres e hijos, han llegado a los Estados Unidos.

\section{Gráfico № 5 . Total de homicidios en países del TNAC, periodo} 1999-2015

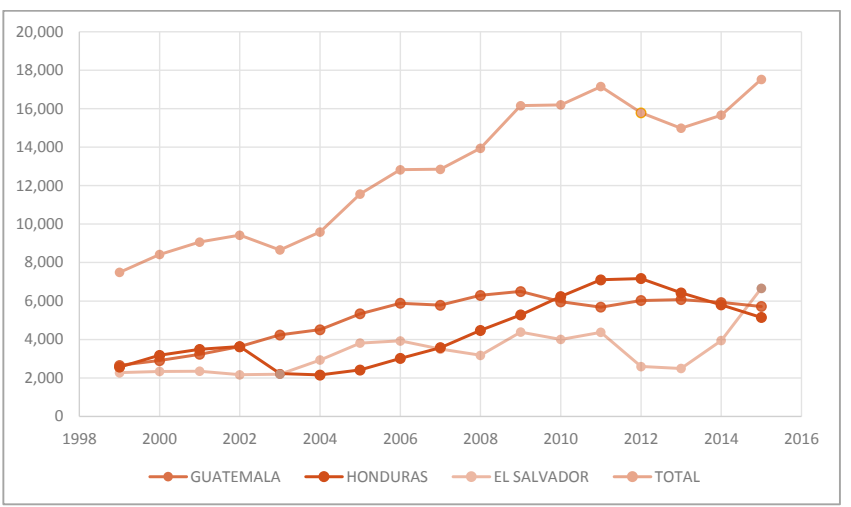

Fuente. Elaboración propia en base a datos INACIF (Guatemala), COEPOL (Honduras), IML (El Salvador)

\section{Conclusiones}

El Salvador, Honduras, y Guatemala están clasificados como uno de los países en el mundo con las tasas más altas de homicidio, estas muertes incluyen hombres, mujeres, niñas y niños.

En 2015, Policía y organismos forenses reportaron una tasa de 104 homicidios por cada 100 mil habitantes en El Salvador, 57 por cada 100 mil habitantes en Honduras y 35.4 por cada 100 mil habitantes en Guatemala. En los tres casos, las cifras sobrepasan el promedio mundial de 6.2 por cada 100 mil habitantes estipulado por la Organización Mundial de la Salud. Además la probabilidad que una de cada 50 personas asesinada muera antes de los 31 años es muy alta.

D Las maras y pandillas y actividades de narcotráfico constituyen factores que contribuyen al incremento de la violencia al servicio al tráfico de drogas y de personas que contratan sus servicios como sicarios, distribución y venta de droga, lo que ha provocado el aumento del consumo local y de la conflictividad social en los tres países.

- Unido a problema de violencia en la región, la pobreza que vive gran parte de la población de estos países tiene un impacto negativo en los países de TNCA pues la falta de fuentes de empleo, acceso a las oportunidades educativas, servicios de salud incrementa la desigualdad social entre la población.

La aplicación disfuncional de las leyes en los piases del
Triángulo Norte ha contribuido a elevar el nivel de crimen y violencia. La impunidad es permanente en esta región: La mayoría de los crímenes no son resueltos por las autoridades competentes. La inhabilidad de las cortes de administrar la justicia y proteger los derechos más fundamentales sirve como un impedimento contra la inversión en la región. Más allá de las percepciones de negligencia o ineptitud, la población del Triángulo Norte tiene motivos para temer que las mismas autoridades que se supone que deben protegerla sean cómplices de la delincuencia organizada o son responsables de abusos ellas mismas.

\section{Bibliografía}

M Amnistía Internacional, octubre, 2016. El papel de Honduras, Guatemala y El Salvador en la creciente crisis de refugiados.

M Centroamérica en cifras. Datos de Seguridad Alimentaria nutricional y Agricultura Familiar. Diciembre de 2011. Págs. 5,6.

\section{CEPAL. Panorámica Social de América Latina.2014}

- Claudio Alberto Dávila-Cervantes, Ana Melisa Pardo-Montaño Análisis de la tendencia de la mortalidad por homicidios en México entre 2000 y 2010.

Mlores Fonseca M.A, (2016); Migración del Triángulo Norte de Centroamérica a los Estados Unidos de América. Revista de Población y Desarrollo. Argonautas y Caminantes, Vol. 12,2016.

M Friedrich Ebert Stiftung. Anuario 2015 de la seguridad regional en América Latina y el Caribe.

http://asjhonduras.com/webhn/tag/triangulo-norte-centroamerica/. (s.f.).

http://www.cepal.org/es/temas/proyecciones-demograficas/estimaciones-proyecciones-poblacion-total-urbana-rural-economicamente-activa

http://elordenmundial.com/2016/09/22/estados-unidos-triangulo-norte-centroamerica-plan-reducir-la-migracion/. (s.f.).

http://internacional.elpais.com/internacional/2012/02/19/actualidad/1329673973_431221.html. (s.f.).

http://www.elheraldo.hn/inicio/916956-465/centroam\%C3\%A9rica-17422-homicidios-en-tri\%C3\%A1ngulo-norte-en-2015. (s.f.). 
http://www.elmundo.es/america/2012/09/27/noticias/1348764878.html. (s.f.).

http://www.estadonacion.or.cr/erca2016/assets/erca2016-web.pdf. (s.f.).

http://www.ibnanalytics.com/asuntos-internacionales/ fuerza-trinacional-para-el-triangulo-del-norte/. (s.f.).

http://www.impactony.com/huyendo-de-la-violencia/. (s.f.).

http://www.laprensa.com.ni/2016/01/05/internacionales/1964380-triangulo-norte-centroamericano-suma-17422. (s.f.).

http://www.laprensagrafica.com/2015/01/11/el-salvador-con-menos-homicidios-en-c-a-pero-con-tendencia-alcista. (s.f.).

http://www.laprensagrafica.com/2016/01/28/exodoy-homicidios-de-jovenes-en-el-triangulo-norte-de-c-a\#sthash.ssrekdml.dpuf. (s.f.).

http://www.laprensagrafica.com/2016/08/29/desplazamiento-forzado-un-asunto-invisible-en-trianguIo-norte-de-centroamerica\#sthash.UAI3LZ6X.dpuf. (s.f.).

http://www.nacion.com/mundo/centroamerica/ Capitales-homicidio-Triangulo-Norte-Centroamerica_0_1591240949.html. (s.f.).

http://www.nacion.com/mundo/centroamerica/ Homicidios-Centroamerica-duplicaron-pese-policias_0_1353064713.html. (s.f.). 